\title{
Akupunktur für den Boden? Direct Push - mit Nadelstichen dem Untergrund auf der Spur
}

\author{
Carsten Leven • Peter Dietrich
}

Online veröffentlicht: 22.12 .2011

(C) Springer-Verlag 2011

Immer wieder schaffen es englische Begriffe im deutschen Sprachraum Einzug zu halten. „Direct Push“ ist einer davon, und bestimmt fragen sich viele Fachkollegen, ob es wirklich eine neue Methode oder ein neuer Begriff für eine alte Sache ist. Die US-amerikanische Umweltbehörde prägte Mitte der 1990er diesen Begriff, unter dem sie ganz allgemein Sondiertechniken für die Erkundung des unverfestigten Untergrundes zusammenfasste. Durch die Verwendung des englischen Begriffs soll dieser Definition Rechnung getragen, aber auch betont werden, dass diese Techniken „direkte“ Messungen von Zustandsgrößen und die „direkte“ Entnahme von Proben während der Sondierung ermöglichen. Damit wird ein wesentlich breiteres Methoden- und Einsatzspektrum als z. B. mit Rammkernsondierungen abgedeckt. Durch ihre Arbeitsweise erlauben Direct-Push-Techniken, mit sehr hoher Auflösung und Verlässlichkeit Untergrunddaten zu erheben. Die Methode ist damit gleichermaßen wissenschaftlich und für die Praxis geeignet und findet immer mehr Anwendungen in der Erkundung von Baugrund, Altlasten sowie bei wasserwirtschaftlichen Fragestellungen.

Für dieses Themenheft haben wir aktuelle Beispiele zusammengetragen, die besonders den Nutzen und die Zweckmäßigkeit der Anwendung zeigen (Martac et al., Radny et al.). Meist ist für die Anwendbarkeit aber eine behörd-

C. Leven $(\bowtie)$

Zentrum für Angewandte Geowissenschaften,

Universität Tübingen,

Hölderlinstr. 12, 72076 Tübingen, Deutschland

E-Mail: carsten.leven-pfister@uni-tuebingen.de

P. Dietrich

Dept. Monitoring- und Erkundungstechnologien,

Helmholtz-Zentrum für Umweltforschung - UFZ,

Permoserstr. 15, 04318 Leipzig, Deutschland

E-Mail: peter.dietrich@ufz.de liche Akzeptanz erforderlich, vor allem wenn es um die Erkundung von Altlasten geht. Diesem Aspekt widmet sich der Beitrag von Wolff. Schließlich zeigt der Beitrag von Liu et al. das Methodenspektrum, mit dem über Direct Push die Verteilung der hydraulischen Durchlässigkeit (dem wichtigsten Parameter der meisten hydrogeologischen Fragestellungen) bestimmt werden kann.

Wir hoffen mit diesem Themenheft die sozusagen ,,angelehnte Tür" für Direct Push weiter aufzustoßen, um deren Akzeptanz und Einsatz zu erweitern. Hierbei ist zu betonen, dass die Anwendung nicht nur spezialisierten Technikanbietern vorbehalten ist. So lassen sich zahlreiche Sondierwerkzeuge an einfache Sondierausrüstungen anpassen, wie sie in der Praxis für die klassische Rammkernsondierung bereits verwendet werden. Beispiele hierzu sind hochaufgelöste Grundwasserbeprobungen, die Bestimmung der hydraulischen Durchlässigkeit oder die der Lithologie über einfache geophysikalische Messungen. Um jedoch die Qualität der erhobenen Daten sicherzustellen und die Wahl der passenden Direct-Push-Werkzeuge zu erleichtern, ist generell die Definition von überprüf- und messbaren Anwendungskriterien erforderlich, was ein weites Feld für zukünftige Arbeiten ist.

Wir hoffen noch viele Beiträge in künftigen Grundwasser-Ausgaben zum Thema Direct Push zu finden und dass dieses Themenheft hierfür als „Appetizer" dient (schon wieder so ein Begriff)... 